\title{
ÉTUDE COMPARATIVE \\ DE DIVERSES MÉTHODES DE DÉTERMINATION DU TAUX DE FOISONNEMENT DES CRÈMES GLACÉES
}

\author{
par \\ J. PITRE et J. OBATON (1)
}

\section{Introduction}

Les crèmes glacées du commerce sont constituées par une émulsion d'air dans le mélange de base soumis à la congélation. Le congélateur continu est, à l'usine, l'organe qui réalise habituellement cette émulsion, tout en abaissant la température du produit (vers $-5^{\circ} \mathrm{C},-7^{\circ} \mathrm{C}$ ) pour qu'il soit ensuite stable le temps $\mathrm{du}$ conditionnement et du durcissement. On obtient à la sortie de cet appareil une consistance plus ou moins pâteuse, résultant du fait qu'une partie de l'eau ( $1 / 3$ à $1 / 2)$ est désormais à l'état de cristaux.

Cette émulsion présente l'intérêt d'améliorer la texture de la crème glacée. De plus l'industriel, qui vend, jusqu'à nouvel ordre, sa production au volume et non au poids, a intérêt à réaliser un taux de foisonnement élevé, et c'est pourquoi en France le déeret du 29 mars 1949, article 6, troisième alinéa, le limite à 100 p. 100. "Le volume du produit prêt à la consommation ne devra pas être supérieur à deux fois le volume total initial des différents constituants ».

On appelle coefficient de foisonnement, le nombre, supérieur à l'unité, par lequel il faut multiplier le volume $V_{1}$ du produit initial pour obtenir le volume $V_{2}$ du produit final, sans variation de la masse $m$ du produit considéré :

$$
V_{2}=V_{1} \cdot f \quad \text { d'où : } \quad f=\frac{V_{2}}{V_{1}}
$$

or : $V_{1}=\frac{m}{\rho_{1}}$, $\rho_{1}$ étant la masse volumique du mélange initial).

et : $\quad V_{2}=\frac{m}{\rho_{2}}, \rho_{2}$ étant la masse volumique du mélange final).

Par suite, la première formule devient :

$$
\frac{m}{\rho_{2}}=\frac{m}{\rho_{1}} \cdot f \quad \text { d'où : } \quad f=\frac{\rho_{1}}{\rho_{2}}
$$

On peut donc connaitre le coefficient de foisonnement en déterminant la masse volumique du produit brut et du produit

(1) Laboratoire départemental et régional de Biologie et d'Hygiène. Caen. 
foisonné. Toutes les méthodes de détermination du coefficient de foisonnement utilisent ce fait. Le taux de foisonnement est le pourcentage d'accroissement de volume d'une masse donnée de crème :

$$
f=\frac{V_{2}-V_{1}}{V_{1}} \times 100 \quad \text { ou : } \quad\left(\frac{\rho_{1}}{\rho_{2}}-1\right) \times 100
$$

A un coefficient de foisonnement de 1,75 par exemple correspond un taux de foisonnement de 75 p. 100. Cela signifie que 1 litre de mélange de base donne 1,75 litre de crème glacée commercialisée, abstraction faite des pertes de substance.

La détermination du taux de foisonnement présente de nombreuses difficultés en raison des faits suivants :

La fusion de la crème glacée le diminue très fortement et le rend instable.

Toute pénétration dans la masse, en vue du prélèvement, ou toute pression, en vue d'introduire le prélèvement dans un récipient, le diminuent par compression.

Il s'agit d'un produit dur et cassant à la température de garde, pâteux à la température la plus fréquente de commercialisation, dont il est de toute façon impossible de remplir correctement un récipient quelconque sans inclure des poches d'air qui viendront augmenter artificiellement le taux de foisonnement mesuré par l'intermédiaire de la masse volumique apparente du produit.

Il est nécessaire de connaître la masse volumique du produit initial. A défaut de pouvoir en effectuer un prélèvement au stade de la fabrication, il faudra se contenter d'une méthode d'appréciation imprécise, soit à partir de la détermination de l'extrait sec, soit par pycnométrie du produit fondu et défoisonné sous vide (opération lente).

Toute modification de température fait varier le foisonnement, car il intervient sur la masse volumique des constituants et sur le pourcentage d'eau à l'état de glace. Mais ceci n'entraîne que des variations inférieures à la limite de précision des méthodes d'évaluation du taux de foisonnement. Les gaz occlus dans la masse solide ne peuvent en effet subir les variations de volume prévisibles d'après la loi de la dilatation des gaz.

\section{I. - Détermination de la masse volumique du produit de base (non foisonné)}

a) Principe du contrôle en fabrication:

Il suffit d'effectuer un prélèvement de crème dans le maturateur en vue d'une détermination pycnométrique. C'est la méthode qu'emploiera le technicien de l'usine chargé du contrôle des fabrications. 
b) Principe du contrôle au stade commercial :

1) Détermination de la matière sèche d'une masse connue d'échantillon (donc abstraction faite de son état physique), et recours à l'hypothèse parfois utilisée selon laquelle la masse volumique de la crème de base serait sensiblement la même que celle d'un sirop de sucre ayant une teneur en saccharose correspondant à la même matière sèche (au moyen d'une table de densité des sirops de sucre).

2) Détermination de l'extrait sec total et de la matière grasse, donc de l'extrait sec dégraissé, en vue d'appliquer la formule approximative suivante, reproduite par J. Jacquet et $R$. Thevenot [1] à partir d'un ouvrage anglais [2] :

Masse volumique du mélange $=$

100

$\frac{\text { p. } 100 \text { matières grasses }}{0,93}+\frac{p .100 \text { extrait sec dégraissé }}{1,58}+\frac{p .100 \text { eau }}{1,58}$

Les méthodes 1 et 2 sont ainsi réalisées :

On remplit de crème glacée un flacon, à ras bord sans inclure d'air. Au laboratoire, on pèse le contenu par la différence de poids entre le flacon plein et le flacon vide lavé et séché.

On connaît ainsi : $\mathrm{Pg}$ le poids de glace et $\mathrm{V}$ le volume du flacon. On détermine $V_{\mathrm{g}}$ le volume de la glace, air déduit, par la formule $\mathrm{V}_{\mathrm{g}}=\mathrm{P}_{\mathrm{g}} / \mathrm{d}$; $\mathrm{d}$ est soit estimé égal à la densité d'un sirop de saccharose ayant la même concentration que la teneur en matière sèche de la glace (1), soit calculé par la formule (2) ; ce dernier procédé nous a donné les meilleurs résultats :

$$
\mathrm{F}=\frac{\mathrm{V}}{\mathrm{V}_{\mathrm{g}}}=\frac{\mathrm{V} \cdot \mathrm{d}}{\mathrm{P}_{\mathrm{g}}}
$$

La particularité de ces deux méthodes réside dans le mode d'estimation de la masse volumique du mélange de base. La deuxième partie, mesure de la masse volumique du mélange foisonné, est faite comme en II-al.

3) Détermination de la masse volumique de la crème après l'avoir soumise à décongélation et défoisonnement sous vide. Un peu lente, cette opération donne néanmoins d'excellents résultats (comparer pour les produits $\mathrm{J}$ et $\mathrm{K}$ les résultats ainsi obtenus avec ceux de la même détermination sur la crème de base non encore traitée). Introduire la crème dans une fiole jaugée de $50 \mathrm{ml}$, et comparer à $20^{\circ} \mathrm{C}$ la masse à celle du même volume d'eau. Bien que les résultats soient d'une excellente reproductibilité, nous avons fait toujours la mayenne de deux déterminations simultanées, comme d'ailleurs pour tous les chiffres avancés dans ce travail.

Lorsque la méthode (a) peut être appliquée, elle doit être considérée comme la meilleure, sous réserve que le prélèvement de la 
crème soit effectué en aval de toute manipulation susceptible d'entraîner une diminution de la masse volumique telle par exemple que le refoulement par de l'eau dans les conduites. A défaut, la méthode 3 servira de référence. Elle donne d'ailleurs des résulțats du même ordre de grandeur.

Le calcul de la masse volumique à partir de la matière sèche donne un résultat sensiblement supérieur à la réalité $(+5,4$ p. 100 en moyenne), sauf pour l'échantillon de glace à la fraise sans produits laitiers, qui se rapproche fortement d'un simple sirop de sucre.

L'introduction d'un facteur correctif tenant compte de la matière grasse diminue considérablement l'erreur qui n'est plus que de 0,10 p. 100 . Dans ces conditions la méthode 2 est séduisante par sa commodité : simple calcul à partir de deux valeurs qui sont déterminées dans toute analyse de conformité à la réglementation.

c) Résultats expérimentaux :

Masse volumique à $20^{\circ} \mathrm{C}$ par rapport à l'eau de la crème brute non foisonnée. Détermination par les méthodes décrites dans le chapitre précédent au paragraphe b).

\section{II. - Détermination de la masse volumique de la crème glacée dans sa présentation commerciale (foisonnée)}

Il faut maintenant déterminer la masse d'un volume connu de substance foisonnée, ou le volume oceupé par une masse connue.

a) Prélèvement d'un volume connu :

1) Remplissage d'un pot taré, jusqu'à ras bord, sans inclure d'air autre que celui émulsionné. Après pesée, remplacement par de l'eau pour mesure de la capacité. Les résultats figurent sur la troisième ligne du tableau II.

Si cette méthode est d'un emploi difficile en présence du produit fini, solide ou semi-solide, elle est utilisable à l'usine à la sortie du congélateur continu, moyennant la précaution de ne pas emprisonner d'air sous la masse pâteuse recueillie directement au bec distributeur, ce qui suppose l'emploi d'un flacon à très large ouverture, dont la surface est difficile à dresser (Résultat lre ligne, tableau II). Mais cette mesure ne tenant pas compte de la variation de volume au cours de la surgélation, les pots remplis comme précédemment ont été placés pleins de crème dans la chambre frigorifique de l'usine où avaient été faits les prélèvements et ressortis au bout d'un temps normal. La surface a alors été dressée à l'aide d'une lame (elle était devenue toujours légèrement convexe) avant pesée. Les résultats figurent sur la deuxième ligne du tableau II. Cette méthode, théoriquement séduisante s'est montrée d'une réalisation très difficile et nous ne pouvons la préconiser. 
TABLEAU I

\begin{tabular}{|c|c|c|c|c|}
\hline Produit & 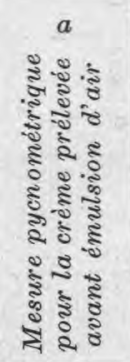 & 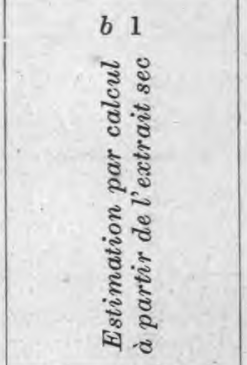 & 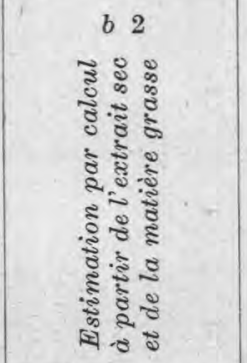 & 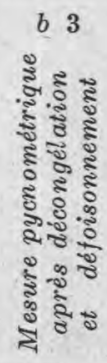 \\
\hline $\begin{array}{l}\text { a) Crème glacée légè- } \\
\text { rement parfumée à la } \\
\text { vanille entrant dans } \\
\text { une préparation com- } \\
\text { plexe }\end{array}$ & 1,086 & $\begin{array}{c}1,1764 \\
\text { (M. S. } 39,96 \% \text { ) }\end{array}$ & (M. G. $16,12 \%$ ) & \\
\hline $\begin{array}{l}\text { b) Crème glacée à la } \\
\text { noisette en purée }\end{array}$ & 1,100 & $\begin{array}{c}1,1828 \\
\text { (M. S. } 41,23 \% \text { ) }\end{array}$ & (M. G. $\quad 6,98 \%$ ) & \\
\hline $\begin{array}{l}\text { c) Crème au café pour } \\
\text { sucettes glacées }\end{array}$ & 1,1065 & $\begin{array}{c}1,1542 \\
\text { (M. S. } 35,60 \% \text { ) }\end{array}$ & (M. G. $8,18 \%$ ) & \\
\hline $\begin{array}{l}\text { d) Crème glacée au } \\
\text { café en coupes }\end{array}$ & & $\begin{array}{c}1,1845 \\
\text { (M.S. } 41,56 \%)\end{array}$ & $\begin{array}{l}\text { 1,110 } \\
\text { (M. G. } 11,98 \% \text { ) }\end{array}$ & 1,1045 \\
\hline $\begin{array}{l}\text { e) Crème glacée au cho- } \\
\text { colat emballée par } \\
\text { tranches }\end{array}$ & & $\begin{array}{c}1,2062 \\
\text { (M. S. } 45,68 \% \text { ) }\end{array}$ & $\begin{array}{c}1,1635 \\
\text { (M. G. } 6,13 \% \text { ) }\end{array}$ & 1,1433 \\
\hline $\begin{array}{l}\text { f) Crème glacée au cho- } \\
\text { colat, en grosse unité }\end{array}$ & & $\begin{array}{c}1,1657 \\
\text { (M. S. } 37,88 \% \text { ) }\end{array}$ & $\begin{array}{c}1,110 \\
\text { (M. G. } 8,95 \%)\end{array}$ & 1,1065 \\
\hline $\begin{array}{l}\text { g) Crème glacée légè- } \\
\text { rement parfumée à la } \\
\text { vanille en grosse } \\
\text { unité }\end{array}$ & & $\begin{array}{c}1,673 \\
\text { (M. S. } 38,21 \% \text { ) }\end{array}$ & $\begin{array}{c}1,1044 \\
\text { (M. G. } 10,33 \%)\end{array}$ & 1,1041 \\
\hline $\begin{array}{l}\text { h) Crème glacée à la } \\
\text { vanille, en grosse } \\
\text { unité }\end{array}$ & & $\begin{array}{c}1,1600 \\
\text { (M. S. } 36,77 \% \text { ) }\end{array}$ & $\begin{array}{c}1,099 \\
\text { (M. G. } 10,06 \%)\end{array}$ & 1,1074 \\
\hline $\begin{array}{l}\text { i) Glace à la fraise, en } \\
\text { grosse unité }\end{array}$ & & $\begin{array}{c}1,1566 \\
\text { (M. S. } 36,08 \% \text { ) }\end{array}$ & $\begin{array}{r}1,152 \\
\text { (M. G. } 0 \% \text { ) }\end{array}$ & 1,1535 \\
\hline $\begin{array}{l}\text { j) Crème glacée à la } \\
\text { vanille en grosse } \\
\text { unité }\end{array}$ & $1,108\left(^{1}\right)$ & $\begin{array}{c}1,1681 \\
\text { (M. S. } 38,36 \% \text { ) }\end{array}$ & $\begin{array}{c}1,1206 \\
\text { (M. G. } 7,50 \%)\end{array}$ & 1,1096 \\
\hline $\begin{array}{l}\text { k) Crème glacée au cho } \\
\text { colat en grosse unité }\end{array}$ & $1,140\left(^{1}\right)$ & $\begin{array}{c}1,1713 \\
\text { (M. S. } 39,04 \% \text { ) }\end{array}$ & $\begin{array}{c}1,131 \\
\text { (M. G. } 6,22 \% \text { ) }\end{array}$ & 1,1368 \\
\hline
\end{tabular}

(1) Déterminations faites par le laboratoire de l'usine. 
TABLEAU II

d) Résultats expérimentaux

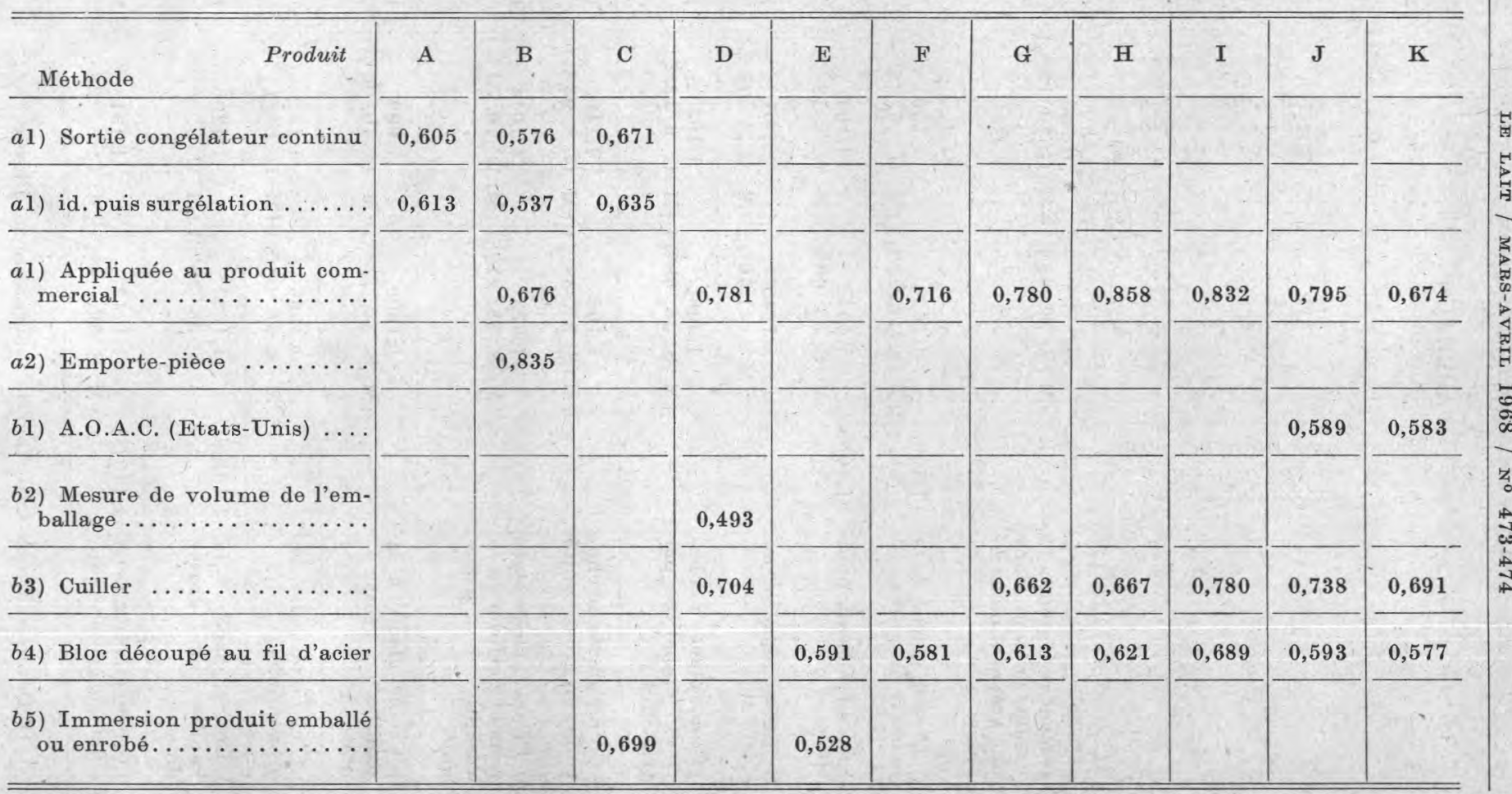


2) Prélèvement à l'emporte-pièce (4 ${ }^{e}$ colonne) : Nous avons essayé d'introduire dans une crème glacée d'un certain volume un cylindre mince ouvert aux deux extrémités, coupant à sa partie inférieure, et pouvant être suspendu à sa partie supérieure. Après pénétration totale, l'appareil est retiré et immédiatement suspendu au fléau d'une balance pour pesée.

Cette méthode n'a pas été suivie, car elle présente un vice rédhibitoire: la viscosité de la crème glacée le long des parois de l'emporte-pièces freine la pénétration et entraîne une compression notable du produit à l'intérieur de l'outil et sous celui-ci. Le niveau de la crème glacée est, en fin de pénétration, beaucoup plus bas dans le cylindre que dans la masse environnante. Les masses volumiques ainsi trouvées sont par suite excessives, et les taux de foisonnement correspondants inférieurs à la réalité.

b) Prélèvement d'une quantité quelconque que l'on pèse, et dont on mesure le volume par l'intermédiaire d'un volume identique de liquide :

Il faut admettre ici une entorse aux règles de mesure physiques : pour éviter une décongélation trop rapide, le liquide en contact avec la crème glacée sera à $+4^{\circ} \mathrm{C}$ et non à $+20^{\circ} \mathrm{C}$. De plus, on assimilera le volume déplacé à celui d'un produit qui est luimême à une température très inférieure à $0^{\circ} \mathrm{C}$. L'imprécision des méthodes, qui ressort des écarts observés entre répétitions, rend illusoire tout calcul de correction.

\section{1) Méthode préconisée par l'A.O.A.C. (3)}

Immersion du prélèvement pesé, dans un bac de kérosène à $+4^{\circ} \mathrm{C}$, jusqu'au ras d'une tubulure de débordement, et pesée du volume de kérosène (de masse volumique préalablement déterminée) déplacé. Cette méthode n'est appliquée aux Etats-Unis qu'à des produits commerciaux entiers d'un volume suffisant. Le kérosène présente par rapport à l'eau l'avantage d'une masse volumique plus faible, d'où résulte une poussée moindre. D'autre part la crème congelée s'y dissout beaucoup moins que dans l'eau.

Nos essais, dont les résultats sont consignés sur la ligne $5 \mathrm{du}$ tableau II, ont été effectués avec du "Kerdane ", de masse volumique 0,7938 à $+4^{\circ} \mathrm{C}$, par rapport à l'eau à la même température. Nous avons utilisé non des produits commerciaux entiers, mais des morceaux de 100 à $125 \mathrm{~g}$ détachés au moyen d'un fil d'acier (type "fil à couper le beurre ") d'une extrémité de produits conditionnés par 1 litre, et déposés sur des feuilles d'aluminium de 0, $200 \mathrm{~g}$. Il est tenu compte dans les calculs des $0,2 \mathrm{ml}$ déplacés par ce papier et le petit dispositif de fil de fer servant à maintenir le prélèvement immergé le temps voulu.

2) Méthode de substitution d'eau dans l'emballage, éventuellement rendu imperméable par paraffinage. L'emballage est pesé 
successivement plein de crème glacée, puis vide. Nous avons. utilisé cette méthode uniquement pour des coupes en carton imperméabilisé. Nous savons d'autre part que des usines l'utilisent pour des emballages en carton d'unités plus volumineuses. Cette méthode s'est révélée mauvaise, ear des poches d'air sont fréquem. ment emprisonnées sous la pâte lors du remplissage, et il est abusif de les compter comme gaz inclus dans la masse par foisonnement (résultat ligne 6, produit D).

3) Méthode de prélèvement d'échantillon à la cuillère. C'est une méthode qui présente l'avantage d'être applicable dans tous les cas, mais l'inconvénient d'être influencée par des effets de compression.

Les échantillons sont disposés dans un flacon à prélèvements, et rapportés congelés au laboratoire, où ils sont placés à $-20^{\circ} \mathrm{C}$ après pesée exacte et rapide. Ou bien le prélèvement est fait au laboratoire dans les unités prélevées intactes.

A l'aide d'une burette de $100 \mathrm{ml}$ (à la rigueur 50) placée dans une enceinte à $+4^{\circ} \mathrm{C}$, et contenant de l'eau à cette température, on remplit le flacon à ras bord et on note le volume nécessaire. Si le contenu est à température suffisamment basse, il ne se décolle pas du verre avant la fin de l'opération et ne cause par conséquent pas de difficulté de flottaison. D'autre part, il se couvre d'une fine couche de glace qui le protège le temps voulu du mélange avec l'eau. Il y a simplement lieu de faire couler l'eau au début le long du verre et non sur le produit, d'opérer rapidement, et de ne pas réchauffer le flacon avec la main.

Le flacon est ensuite vidé, lavé, séché, pesé. Sa capacité totale est enfin mesurée à l'aide d'eau à $+4^{\circ} \mathrm{C}$, comme précédemment. Les résultats figurent sur la $7^{\mathrm{e}}$ ligne.

4) Chaque fois que cela nous a été possible (échantillons parallélépipédiques d'un volume suffisant ou petites unités entières faciles à déballer et constituées d'une seule crème), nous avons opéré de la même façon, mais en prélevant à l'aide d'un fil d'acier réduisant à très peu de chose les compressions. Le prélèvement peut de plus avoir les cotes maximales compatibles avec celles du flacon, et l'on est en droit de considérer que les résultats ainsi obtenus ( $8^{\mathrm{e}}$ ligne) sont, avec ceux de la méthode américaine, les plus proches de la réalité.

5) Pour certains produits de petites dimensions : sucettes glacées, tranches emballées séparément dans du papier métallisé, nous avons opéré de façon un peu différente :

- Pesée de 2 ou 3 unités, emballages adhérents ou enrobages (et bâtonnets) compris.

- Mesure du volume d'eau à $+4^{\circ} \mathrm{C}$ déplacé, dans une éprouvette appropriée (250 ou $500 \mathrm{ml}$ ) par les mêmes unités. 
- Pesée des emballages ou enrobages (et batonnets) correspondants, lavés et séchés à $+30^{\circ} \mathrm{C}$. Dans de l'eau à $+15^{\circ} \mathrm{C}$, il est facile de séparer l'enrobage de chocolat ferme et de le recueillir intégralement.

- Mesure dans une petite éprouvette du volume des emballages ou de l'enrobage et du batonnet.

- On en déduit la masse et le volume de la crème glacée, donc sa masse volumique (résultats ligne 9). Cette méthode, satisfaisante pour les sucettes glacées, fait un peu double emploi avec la méthode 4 : l'enrobage étant enlevé sans se préoccuper d'un léger entraînement de crème, cette dernière est recueillie dans un flacon, en la détachant d'un bloc de son support. D'autre part, pour les tranches emballées, il a été observé qu'il y avait de l'air emprisonné dans l'emballage, et que le taux de foisonnement s'en trouvait surestimé.

c) Méthode mixte avec mesure successive du volume avant et après décongélation et défoisonnement du même prélèvement placé en éprouvette graduée. Bien que cette méthode n'ait pas été suivie, en raison de l'imprécision des lectures de volume, nous la mentionnons pour le cas où serait trouvée par la suite une modification applicable:

Une éprouvette de $500 \mathrm{ml}$ est percée près de sa base d'un trou de passage d'une tubulure latérale de faible diamètre. Celle-ci est graduée par des apports successifs de $5 \mathrm{ml}$ d'eau dans l'éprouvette. Elle servira à suivre les variations de volume sans que le ménisque soit modifié par la mousse, les corps gras ou l'éther.

Le produit à examiner, fraction ou totalité d'un échantillon commercial, est rapidement immergé dans un volume connu d'eau à $+4^{\circ} \mathrm{C}$ et maintenu sous la surface à l'aide d'un fil de fer léger muni d'un croisillon à son extrémité, le temps nécessaire à la lecture d'augmentation de volume.

On laisse l'échantillon se décongeler. On élimine la mousse par agitation (la dilution dans l'eau facilite cette opération) et l'on termine en versant à la surface quelques gouttes d'éther sulfurique ou en plaçant tout le dispositif.sous une cloche à vide.

Une nouvelle lecture de volume donne par différence le volume des gaz émulsionnés dans le volume initialement mesuré de crème glacée foisonnée.

\section{Calcul du taux de foisonnement:}

1) Les deux premières lignes du tableau précédent ne présentent pas de différences significatives. Leur moyenne doit se rapprocher sensiblement de la réalité.

Cela permet de calculer pour les trois premiers produits le taux de foisonnement en utilisant la colonne a) du premier tableau comme terme de comparaison : 


$$
\begin{aligned}
& F_{A}=\left[\frac{1,086}{0,609}-1\right] 100=78,3 \\
& F_{B}=\left[\frac{1,100}{0,5565}-1\right] 100=97,7 \\
& F_{C}=\left[\frac{1,1065}{0,653}-1\right] 100=69,4
\end{aligned}
$$

Pour le produit B il est possible de comparer ce résultat avec celui obtenu en utilisant la masse volumique du produit foisonné donnée par le $3^{\mathrm{e}}$ et $4^{\mathrm{e}}$ lignes du $2^{\mathrm{e}}$ tableau. On a alors respectivement:

$$
\begin{aligned}
& \mathrm{F}_{\mathrm{B}^{\prime}} \text { d'après a } 1=\left[\frac{1,100}{0,676}-1\right] 100=62,7 \\
& \mathrm{~F}_{\mathrm{B}^{\prime \prime}} \text { d'après a } 2=\left[\frac{1,100}{0,835}-1\right] 100=31,7
\end{aligned}
$$

Les écarts considérables résultent d'une erreur d'appréciation de la masse volumique de la crème glacée foisonnée. Les résultats des lignes a 1, sur produit commercial et a 2 sont ici à rejeter.

Il est possible de comparer de la même façon pour le produit C le taux de foisonnement calculé précédemment à celui qu'on obtient avec le chiffre de la ligne b 5 :

$$
\mathrm{F}_{\mathrm{C}^{\prime}}=\left[\frac{1.1065}{0,699}-1\right] 100=58,3
$$

La précision est médiocre, et cependant dans le cas des sucettes glacées, c'est la méthode la meilleure qu'on puisse appliquer à des prélèvements dans le commerce.

2) 7 autres produits commerciaux ont fait l'objet d'une mesure de masse volumique de la crème foisonnée par la technique a 1 et celle-ci est comparée à la masse volumique de la crème de base, mesurée par pyenométrie.

A côté des taux de foisonnement correspondants, qui apparaissent tous comme trop faibles, nous avons noté les taux obtenus en utilisant d'autres méthodes :

Les méthodes $\mathrm{b} 1, \mathrm{~b} 3$ et $\mathrm{b} 4$ ne présentant aucun défaut qui puisse expliquer une sous-évaluation erronée de la masse volumique des produits foisonnés, donc une sur-évaluation du taux de foisonnement, la valeur trouvée pour ce taux doit être considérée comme d'autant plus exacte qu'elle est plus élevée. Les méthodes b 1 et b 4 apparaissent alors comme les seules valables. La méthode des cuillerées b 3, qui a pourtant l'avantage d'un emploi plus universel, 
est fausse dans des proportions notables par défaut et son emploi doit être limité aux cas où toute autre est inapplicable (échantillons débités à la cuiller dite " américaine »).

\begin{tabular}{|c|c|c|c|c|c|}
\hline Produits & $\begin{array}{ll}a & 1\end{array}$ & $b 1$ & $b 3$ & $b 4$ & $\begin{array}{l}\text { d'après la comp- } \\
\text { tabilité, matières } \\
\text { de l'usine, compte } \\
\text { tenu des pertes }\end{array}$ \\
\hline $\begin{array}{l}\mathrm{D} \\
\mathrm{F} \\
\mathrm{G} \\
\mathrm{H} \\
\mathrm{I} \\
\mathrm{J} \\
\mathrm{K}\end{array}$ & $\begin{array}{l}41,4 \\
54,5 \\
41,6 \\
29,1 \\
38,6 \\
39,5 \\
68,7\end{array}$ & $\begin{array}{l}88,5 \\
95,0\end{array}$ & $\begin{array}{l}56,9 \\
66,8 \\
66,0 \\
47,9 \\
52,3 \\
64,4\end{array}$ & $\begin{array}{l}90,4 \\
80,1 \\
78,4 \\
67,4 \\
87,0 \\
97,0\end{array}$ & $\begin{array}{l}55,2 \\
72,7\end{array}$ \\
\hline
\end{tabular}

Quant à la méthode a 1 , elle se révèle là encore en défaut. De plus elle n'est reproductible qu'à $4,3 \%$ près, d'après les résultats de 4 répétitions simultanées pour les produits $J$ et $K$ alors que la méthode b 1 (A.O.A.C.) ne donne que des écarts de $\pm 0,41$ p. 100 et la méthode b 4 de $\pm 0,54$ p. 100 .

Le bilan de fabrication de l'usine, effectué en poids et non en volume, ne peut en aucune façon nous donner de renseignements exploitables, car pour passer aux volumes, nous devons utiliser les moyennes, établies antérieurement pour des produits similaires de déterminations approchées du volume et du poids unitaires. Pour le produit D en coupes, la méthode des cuillérées est la moins mauvaise de celles que nous avons expérimentées. Il est toutefois évidemment possible d'obtenir un meilleur résultat en déballant l'échantillon (découper et décoller le carton) et en le traitant par l'une des méthodes b 1 ou b 4 avec des résultats de même ordre de précision.

4) Pour le produit E, l'essai de détermination de la masse volumique par pesée et mesure de volume du produit non déballé à donné un résultat imparfait, le taux de foisonnement étant surévalué en raison de l'inclusion de petites poches d'air sous l'emballage métallisé.

$\mathrm{F}_{\mathrm{E}}$ selon b $4 \mathrm{du} 2^{\mathrm{e}}$ tableau $=93,4$ selon $\mathrm{b} 5 \mathrm{du} 2^{\mathrm{e}}$ tableau $=116,5$. 